\title{
Electronic origin of the high thermoelectric performance of GeTe among the p-type group IV monotellurides
}

\author{
Juan $\mathrm{Li}^{1}$, Zhiwei Chen ${ }^{1}$, Xinyue Zhang ${ }^{1}$, Yongxing Sun ${ }^{2}$, Jiong Yang ${ }^{2}$ and Yanzhong Pei ${ }^{1}$
}

$\mathrm{PbTe}$ and $\mathrm{SnTe}$ in their p-type forms have long been considered high-performance thermoelectrics, and both of them largely rely on two valence bands (the first band at $L$ point and the second one along the $\Sigma$ line) participating in the transport properties. This work focuses on the thermoelectric transport properties inherent to p-type GeTe, a member of the group IV monotellurides that is relatively less studied. Approximately $50 \mathrm{GeTe}$ samples have been synthesized with different carrier concentrations spanning from 1 to $20 \times 10^{20} \mathrm{~cm}^{-3}$, enabling an insightful understanding of the electronic transport and a full carrier concentration optimization for the thermoelectric performance. When all of these three monotellurides (PbTe, SnTe and GeTe) are fully optimized in their p-type forms, GeTe shows the highest thermoelectric figure of merit ( $z T$ up to 1.8). This is due to its superior electronic performance, originating from the highly degenerated $\Sigma$ band at the band edge in the low-temperature rhombohedral phase and the smallest effective masses for both the $L$ and $\Sigma$ bands in the high-temperature cubic phase. The high thermoelectric performance of GeTe that is induced by its unique electronic structure not only provides a reference substance for understanding existing research on GeTe but also opens new possibilities for the further improvement of the thermoelectric performance of this material.

NPG Asia Materials (2017) 9, e353; doi:10.1038/am.2017.8; published online 10 March 2017

\section{INTRODUCTION}

Owing to the growing energy crisis, thermoelectric conversion as a clean and sustainable solution has attracted increasing attention because it can directly convert waste heat to electricity. ${ }^{1}$ However, the greatest challenge that prevents its widespread application is its relatively low conversion efficiency, which is evaluated by the TE figure of merit, $z T=\left(S^{2} T\right) /\left(\rho\left(\kappa_{\mathrm{E}}+\kappa_{\mathrm{L}}\right)\right)$, where $S, T, \rho, \kappa_{\mathrm{E}}$ and $\kappa_{\mathrm{L}}$ are the Seebeck coefficient, absolute temperature, electrical resistivity, and electronic and lattice contributions to the thermal conductivity, respectively.

It is commonly recognized that the Seebeck coefficient, resistivity and electronic thermal conductivity are strongly coupled with each other. This led to most of the research efforts in TE being focused on minimizing the lattice thermal conductivity, which is the only independent material parameter. Effective approaches include nanostructuring, ${ }^{2-7}$ lattice anharmonicity, ${ }^{8,9}$ liquid phonons, ${ }^{10,11}$ vacancy $^{12-14}$ or interstitial ${ }^{15}$ point defects and a low sound velocity. ${ }^{16}$ Band engineering concepts including a large number of degenerated bands, ${ }^{17-25}$ a low band effective mass ${ }^{26}$ and weak carrier scattering $^{27}$ have also proven to be successful in enhancing the TE performance. The knowledge of the band structure is thus critical for band engineering and optimizing the electrical transport of TE materials.

P-type PbTe and SnTe have long been studied as TE materials, but GeTe, another member of the group IV monotellurides, has been less studied. $\mathrm{PbTe}^{28-30}$ and $\mathrm{SnTe}^{31-34}$ both have a higher-energy $L$ and a lower-energy $\Sigma$ valence band. In pristine materials with optimized carrier concentrations, the lower-energy offset between these two bands in $\mathrm{PbTe}\left(0.17 \mathrm{eV}^{29,30,35}\right.$ at $\left.300 \mathrm{~K}\right)$ compared with that in SnTe $\left(0.3-0.4 \mathrm{eV}^{32,34}\right.$ at $\left.300 \mathrm{~K}\right)$ leads to a much higher-power factor $\left(30 \mu \mathrm{W} \mathrm{cm}{ }^{-1} \mathrm{~K}^{-236}\right)$ for $\mathrm{PbTe}$ than that for SnTe $\left(20 \mu \mathrm{W} \mathrm{cm}{ }^{-1} \mathrm{~K}^{-237}\right)$. For $\mathrm{GeTe}$, the available literature shows a power factor of $\sim 40 \mu \mathrm{W} \mathrm{cm}{ }^{-1} \mathrm{~K}^{-2}, 38-41$ which is the highest among the three compounds. The underlying origin of this phenomenon, however, is ambiguous, to our best knowledge. The lack of a detailed investigation of GeTe has also hindered the understanding of some GeTe-based alloys with decent TE properties, such as GeTe-AgSbTe (TAGS), ${ }^{42-45}(\mathrm{GeTe})_{n} \mathrm{Sb}_{2} \mathrm{Te}_{3}(\mathrm{GST}){ }^{46-48}\left(\mathrm{Bi}_{2} \mathrm{Te}_{3}\right)_{x}(\mathrm{GeTe})_{1-x}{ }^{49-52}$ and $\mathrm{Ge}_{1-x} \mathrm{~Pb}_{x} \mathrm{Te}^{53-58}$ Therefore, it is of great importance to reveal the essence of the superior electronic performance in GeTe, particularly from the perspective of the electronic structure.

\footnotetext{
${ }^{1}$ Key Laboratory of Advanced Civil Engineering Materials of Ministry of Education, School of Materials Science and Engineering, Tongji University, Shanghai, China and ${ }^{2}$ Materials Genome Institute, Shanghai University, Shanghai, China

Correspondence: Professor Y Pei, Key Laboratory of Advanced Civil Engineering Materials of Ministry of Education, School of Science and Engineering, Tongji University, 4800 Caoan Road, Shanghai 201804, China.

E-mail: yanzhong@tongji.edu.cn

or Professor J Yang, Materials Genome Institute, Shanghai University, 99 Shangda Road, Shanghai 200444, China.

E-mail: jiongy@t.shu.edu.cn

Received 12 September 2016; revised 11 October 2016; accepted 15 November 2016
} 
In addition to the reported high TE performance of GeTe-based materials, its phase transition deforms the lattice of GeTe from a $\mathrm{NaCl}$-type face-centered cubic $(\beta-\mathrm{GeTe})$ structure to a rhombohedral $\left(\alpha\right.$-GeTe) structure ${ }^{59,60}$ at $\sim 700 \mathrm{~K}$ or below. ${ }^{61}$ The subtle changes of the Seebeck coefficient and resistivity in the vicinity of the phase transition temperature have been barely discussed. This phase transition, limited only in GeTe among these three group IV

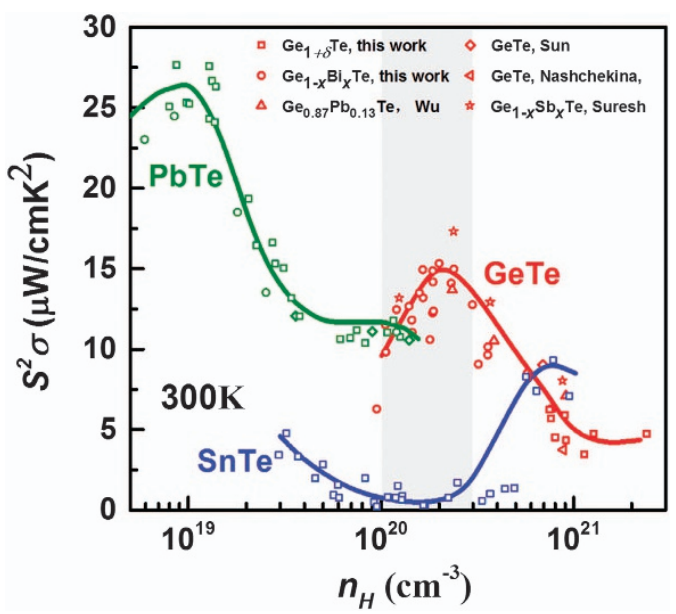

Figure 1 Room-temperature Hall carrier concentration-dependent power factor $\left(P F=S^{2} \sigma\right)$ for $\mathrm{SnTe}_{1-x} x_{x},{ }^{37} \mathrm{Na}_{x} \mathrm{~Pb}_{1-x} \mathrm{Te}^{36}$ and GeTe in comparison with literature data for $\mathrm{p}-\mathrm{GeTe} .^{38-40,88}$ The optimal carrier concentration for GeTe is found to be $1-3 \times 10^{20} \mathrm{~cm}^{-3}$ (shadow area).
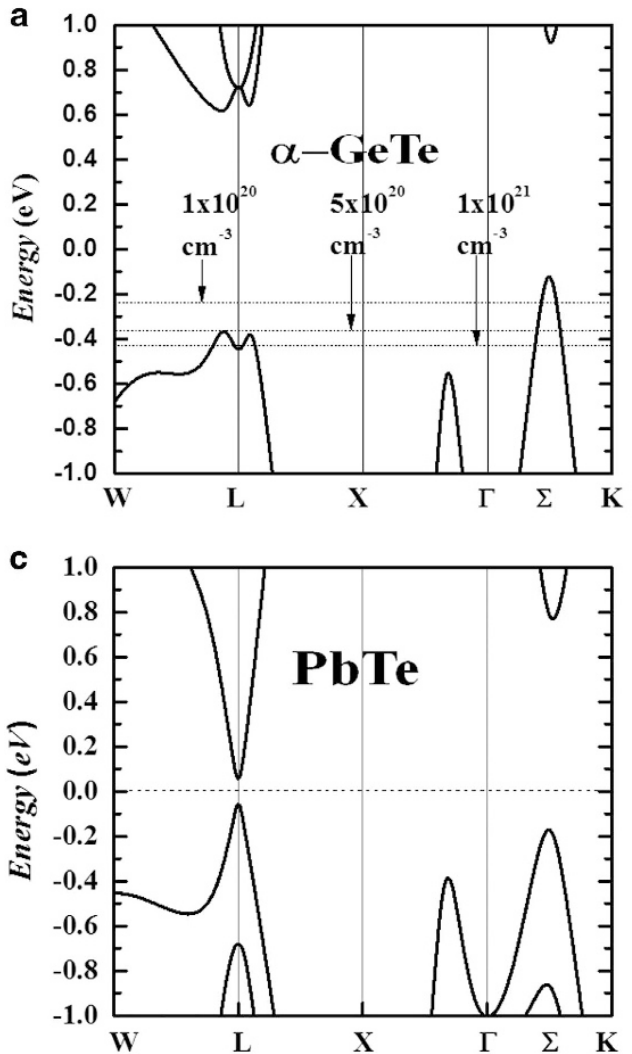

monotellurides, in principle enables one more degree of freedom to tune the transport properties.

Since band engineering approaches such as band convergence have recently proven to enhance effectively the TE performance of both $\mathrm{PbTe}^{17,21,23,62}$ and $\mathrm{SnTe}^{63,64}$ in their p-type forms, it is believed that a detailed investigation of the band structure and the inherent transport properties of GeTe in both its low- and high-temperature phases would be helpful not only for guiding the band/microstructure engineering approaches for further improvements but also for evaluating their net contributions.

In this work, we synthesized and measured $\sim 50$ GeTe samples with a broad range of carrier concentrations and performed first-principles band structure calculations. The transport properties can be well understood based on a rigid band approximation and are well consistent with our calculations. When fully optimized, GeTe surprisingly shows the highest $z T$ (a peak value of 1.8) among the three group IV monotellurides in the entire temperature range. As it is well demonstrated that a high band degeneracy is beneficial for a high power factor, ${ }^{17,62}$ the high $z T$ of GeTe in the low-temperature rhombohedral phase $(\alpha)$ electronically originates from the unique dominance of the conduction by its high-degeneracy $\Sigma$ band (In the Brillouin zone of the rhombohedral phase, the original 8 half valleys of $L$ valence band in the Brillouin zone of the cubic phase is split into 6 half valleys at $L$ point and the rest 2 at $Z$ point, whereas the original 12 full valleys of $\Sigma$ valence band is split into 6 full valleys along $\Sigma$ line and the rest 6 along $\eta$ line.), whereas the conduction of the other two group IV monotellurides is dominated by the low-degeneracy $L$ band. In the high-temperature cubic phase $(\beta)$, the smallest effective masses for both the $L$ and $\Sigma$ bands are responsible for the high power factor
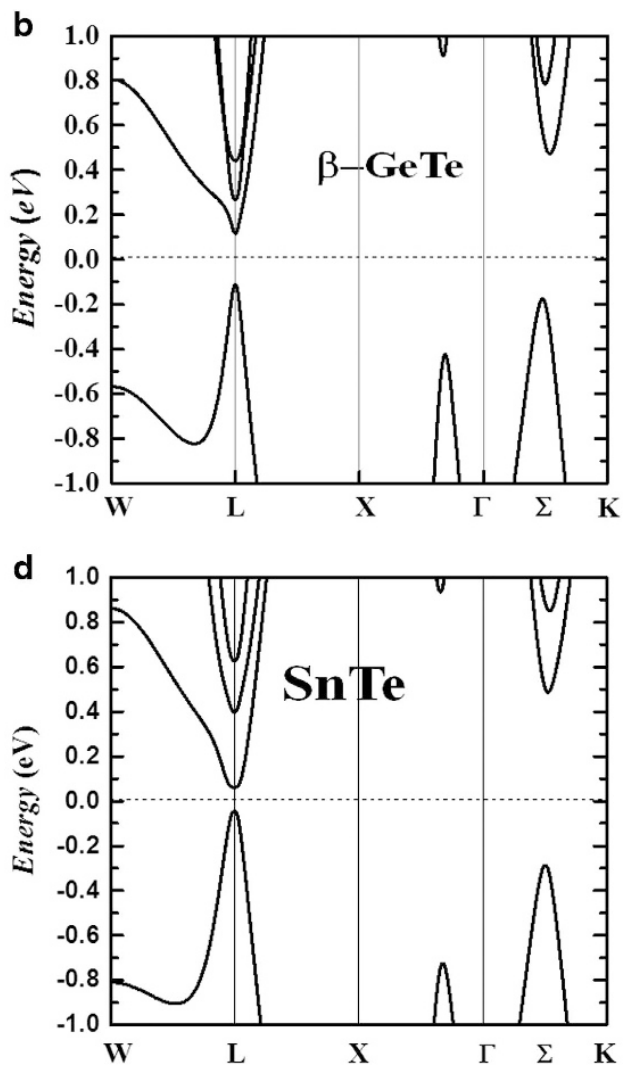

Figure 2 Calculated band structures for GeTe in both rhombohedral (a) and cubic structures (b), PbTe (c) and SnTe (d). A few Fermi levels with their corresponding carrier concentrations are given in (a) for rhombohedral GeTe. 
in GeTe. This work not only demonstrates that the band structure guarantees a high TE performance inherent to GeTe in both the lowand high-temperature phases but it also establishes a reference substance for evaluating the net effects of additional approaches such as band engineering and nanostructuring for possible further improvements of this material.

\section{MATERIALS AND METHODS}

Because of the stoichiometric deviation in $\mathrm{GeTe}$, Ge vacancies ${ }^{65,66}$ cause a very high hole concentration on the order of $10^{21} \mathrm{~cm}^{-3}$, which is significantly higher than the optimal value for this material. Polycrystalline $\mathrm{Ge}_{1-x} \mathrm{Bi}_{x} \mathrm{Te}$ $(x \leqslant 0.1)$ and $\mathrm{Ge}_{1+\delta} \mathrm{Te}(-0.04 \leqslant \delta<0.06)$ samples with controlled carrier concentrations were synthesized by melting stoichiometric amounts of high-purity elements (>99.99\%) in vacuum quartz ampoules at $1073 \mathrm{~K}$ for $6 \mathrm{~h}$, quenching in cold water and annealing at $873 \mathrm{~K}$ for 3 days. The ingots were hand ground into fine powders for X-ray diffraction, and for hot-pressing by induction heating ${ }^{67}$ at $853 \mathrm{~K}$ for $40 \mathrm{~min}$ under a uniaxial pressure of $\sim 80 \mathrm{MPa}$. The obtained dense pellets ( $>98 \%$ of the theoretical density) were $\sim 12 \mathrm{~mm}$ in diameter and $1.5 \mathrm{~mm}$ in thickness for the transport property measurements.

The resistivity, Seebeck coefficient and Hall coefficient were simultaneously measured on the pellet samples, where the hot and cold side temperatures were measured by two K-type thermocouples attached to the opposite edges of the pellet sample and the thermopower was measured by two $\mathrm{Nb}$ wires welded to the thermocouple tips. This technique is quite important because GeTe undergoes a phase transition. The resistivity and Hall coefficient were measured using the Van der Pauw technique under a reversible magnetic field of $1.5 \mathrm{~T}$. The Seebeck coefficient was obtained from the slope of the thermopower vs temperature gradient from 0 to $5 \mathrm{~K}^{68}$ The thermal diffusivity $(D)$ was measured by a laser flash technique (model: LFA-457, Netzsch, Selb, Germany). A Dulong-Petit limit of the heat capacity $\left(C_{\mathrm{p}}\right)$ is used, which is consistent with the measurements ${ }^{40}$ for GeTe alloys and with those in many recent reports ${ }^{47,69,70}$ The thermal conductivity is determined via $\kappa=d C_{\mathrm{p}} D$, where $d$ is

Table 1 Calculated band parameters for GeTe, PbTe and SnTe

\begin{tabular}{|c|c|c|c|c|c|c|c|}
\hline & $\Delta \mathrm{E}_{\mathrm{L}-\Sigma}(\mathrm{eV})$ & $\mathrm{m}_{\left\llcorner\perp^{*}\right.}\left(\mathrm{m}_{0}\right)$ & $m_{L \|}{ }^{*}\left(m_{0}\right)$ & $m_{L}{ }^{*}\left(m_{0}\right)$ & $\mathrm{m} \Sigma_{\perp} *\left(\mathrm{~m}_{0}\right)$ & $\mathrm{m} \Sigma_{\|}{ }^{*}\left(m_{0}\right)$ & $\mathrm{m} \Sigma^{*}\left(\mathrm{~m}_{0}\right)$ \\
\hline$\alpha$-GeTe & -0.174 & 0.044 & 0.426 & 0.094 & 0.182 & 1.085 & 0.330 \\
\hline$\beta$-GeTe & 0.064 & 0.044 & 0.426 & 0.094 & 0.155 & 1.423 & 0.325 \\
\hline SnTe & 0.241 & 0.056 & 0.301 & 0.098 & 0.130 & 6.515 & 0.479 \\
\hline
\end{tabular}

Abbreviations: $\Delta \mathrm{E}_{\mathrm{L}-\Sigma}$, energy offset of $\mathrm{L}$ band and sigma band; $\mathrm{m}_{\perp}{ }^{*}$, effective mass of $\mathrm{L}$ band along perpendicular direction; $\mathrm{m}_{\perp \|^{*}}$, effective mass of $\mathrm{L}$ band along the parallel direction; $m_{L^{*}}$, effective mass of $L$ band; $m \Sigma_{\perp}{ }^{*}$, effective mass of sigma band along the perpendicular band; $m m \Sigma_{\|}^{*}$, effective mass of sigma band along the parallel band; $m \Sigma^{*}$, effective mass $\mathrm{m}_{\mathrm{L}}{ }^{*}$, effective
of sigma band.
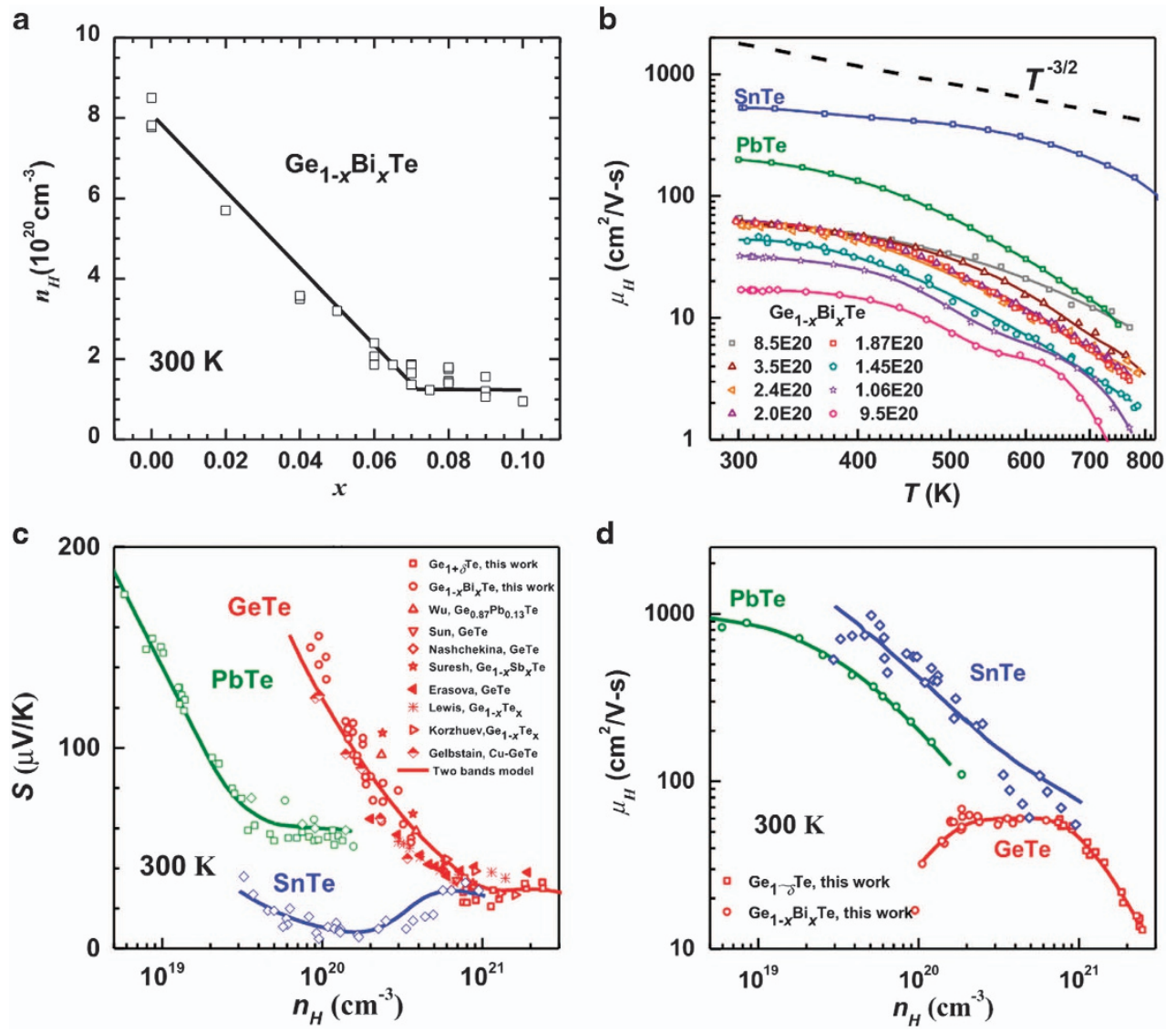

Figure 3 Linear increase of room-temperature Hall carrier concentration with Bi-doping concentration (a) for $\mathrm{Bi}_{x} \mathrm{Ge}_{1-x} \mathrm{Te}$ with $x \leqslant 0.07$ and a saturation with $x>0.07$. Temperature-dependent Hall mobility (b), Hall carrier concentration-dependent Seebeck coefficient (c) and Hall mobility (d) for $\mathrm{SnTe}_{1-x} x_{x},{ }^{37}$ $\mathrm{Na}_{x} \mathrm{~Pb}_{1-x} \mathrm{Te}^{36}{ }^{36} \mathrm{Bi}_{x} \mathrm{Ge}_{1-x} \mathrm{Te}$ and $\mathrm{Ge}_{1+\delta} \mathrm{Te}$ in comparison with literature data on p-GeTe. ${ }^{38-40,51,79,88-90}$ 
the density. All of the transport property measurements were carried out from 300 to $800 \mathrm{~K}$, and the reproducibility is confirmed by multiple measurements

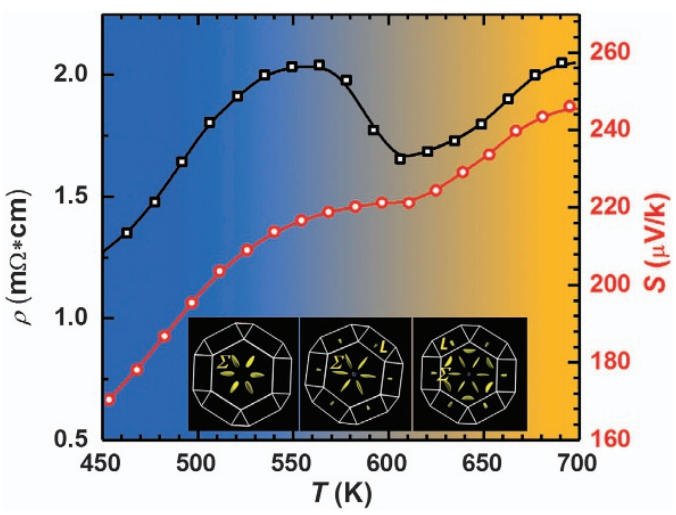

Figure 4 Temperature-dependent Seebeck coefficient and resistivity for $\mathrm{Bi}_{x} \mathrm{Ge}_{1-x} \mathrm{Te}$ with $x=0.075\left(n_{\mathrm{H}}=1.37 \times 10^{20} \mathrm{~cm}^{-3}\right)$ showing a transition from conduction by the $\Sigma$ band in $\alpha$-GeTe at $T<\sim 550 \mathrm{~K}$ to that by both the $L$ and $\Sigma$ bands in $\beta$-GeTe at $T>\sim 625 \mathrm{~K}$ due to the phase transition. The insets show that the Fermi surface evolution is related to the transition. The much larger effective mass of the $\Sigma$ band than that of the $L$ band leads to a decrease in the Seebeck coefficient and resistivity, which is consistent with the band structure calculation showing a switch of the principal valence band from $\Sigma$ to $L$ due to the phase transition.

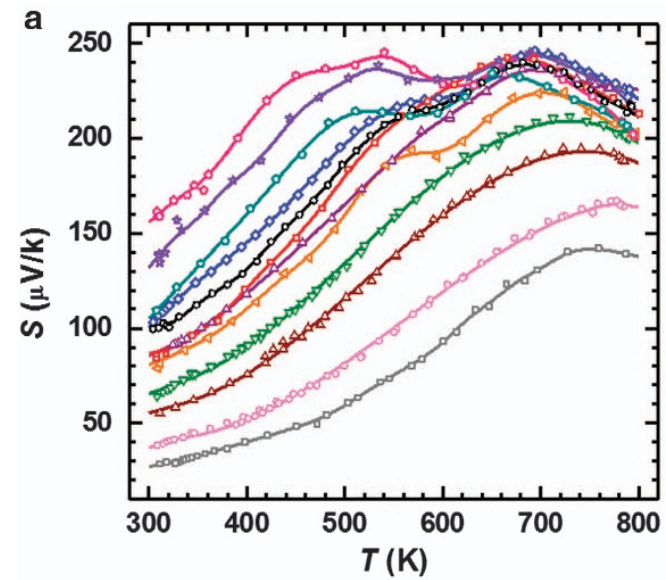

C

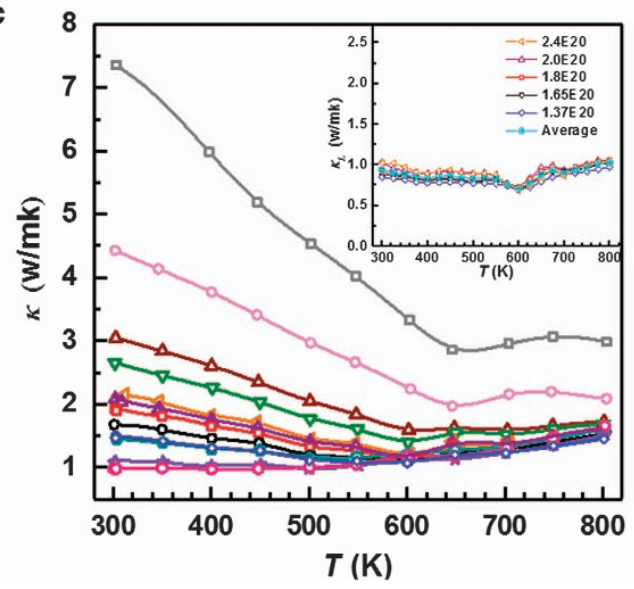

on the same sample and in multiple samples with similar carrier concentrations.

The first-principles calculations on the band structures were performed with the Vienna $a b$ initio simulation package. The generalized gradient approximation functional and the projected augmented wave method were used. The energy cutoff was $300 \mathrm{eV}$, and the spin-orbit coupling was included. For simplicity, the band structure for $\alpha$-GeTe is shown without spin-orbit coupling because the valence band in the vicinity of the Fermi level show no difference other than a weak split for the $\Sigma$ band.

\section{RESULTS AND DISCUSSION}

The X-ray diffraction patterns of $\mathrm{Ge}_{1}-{ }_{x} \mathrm{Bi}_{x} \mathrm{Te}(0 \leqslant x \leqslant 0.1)$ are shown in Supplementary Figure 1. All of the diffraction peaks can be indexed to the rhombohedral structure when $x \leqslant 0.05$. With a further increase in $x$, trace Ge impurity peaks are observable, which is consistent with our scanning electron microscope observations, as shown in Supplementary Figure 2.

The room-temperature carrier concentration-dependent power factor $\left(P F=S^{2} \sigma\right)$ for GeTe is consistent with available literature results, as shown in Figure 1. The optimal carrier concentration for GeTe is found to be $1-3 \times 10^{20} \mathrm{~cm}^{-3}$. The similarity among these three compounds is that they all have a two-valence band structure. The difference can be seen in the calculated band structures for $\alpha$-GeTe, $\beta$-GeTe, PbTe and SnTe, as shown in Figure 2, with the detailed band parameters listed in Table 1. The calculated band structures for GeTe are consistent with those in the available literature. ${ }^{71-79}$ It is seen
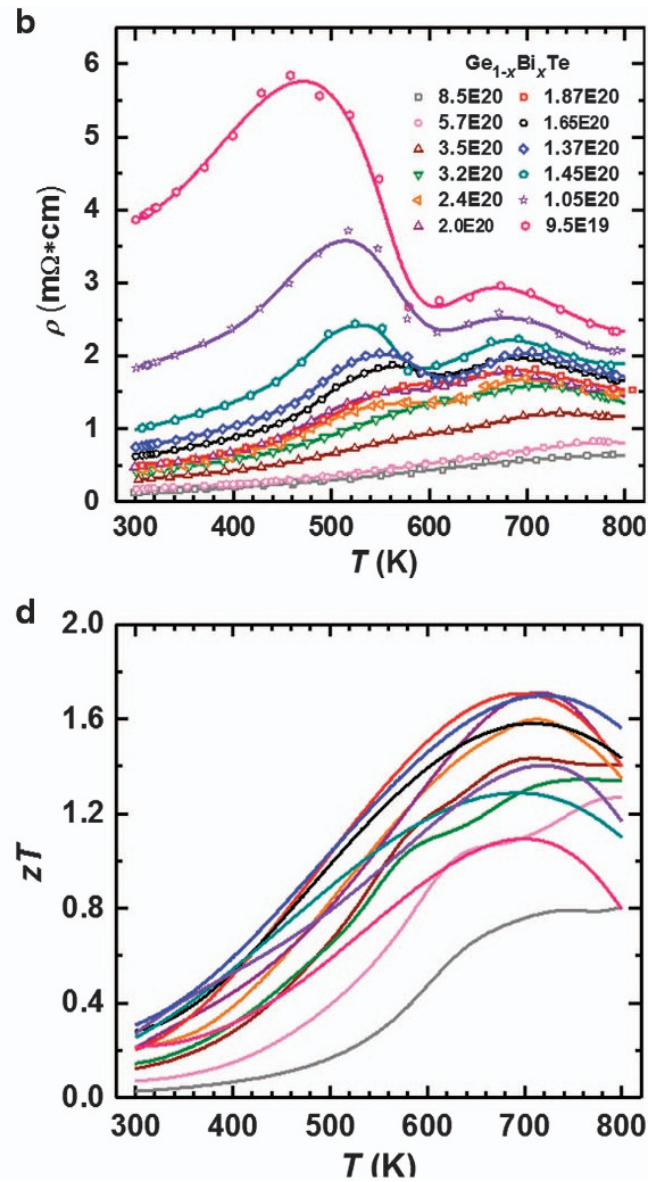

Figure 5 Temperature-dependent resistivity (a), Seebeck coefficient (b), thermal conductivity and figure of merit (d) for $\mathrm{Bi}_{x} \mathrm{Ge}_{1-x} \mathrm{Te}_{\mathrm{e}}$ with room-temperature Hall carrier concentrations ranging from $\sim 10^{20}$ to $10^{21} \mathrm{~cm}^{-3}$. The inset in c shows the lattice thermal conductivity and an average value for a few optimally doped $\left(n_{\mathrm{H}} \sim 2 \pm 0.6 \times 10^{20} \mathrm{~cm}^{-3}\right)$ samples. 
a

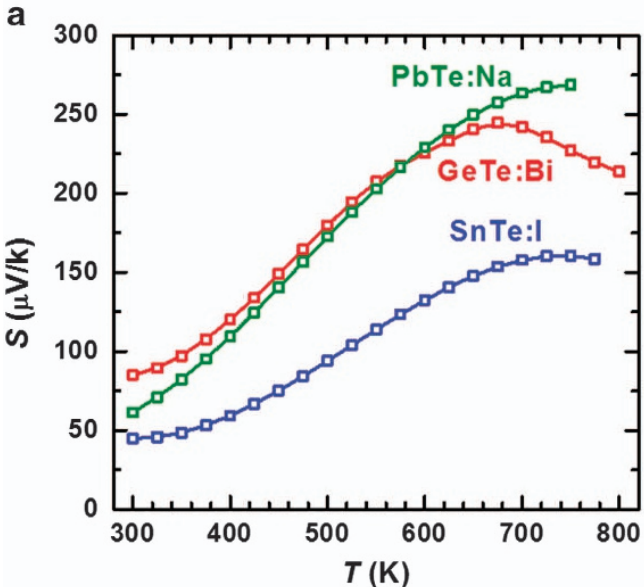

c

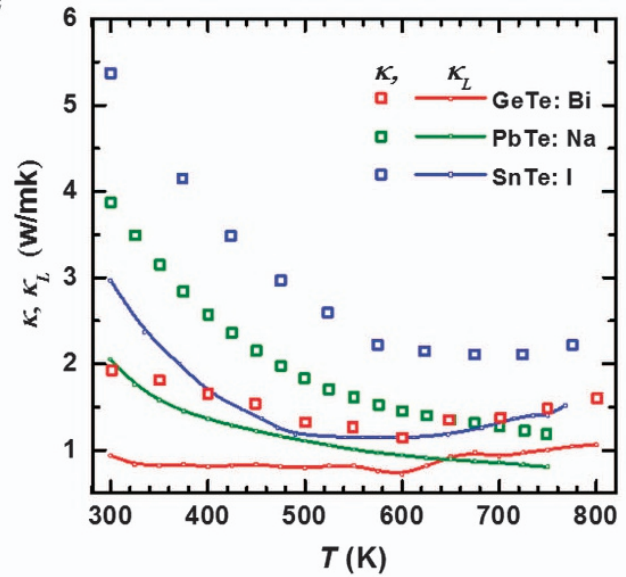

b

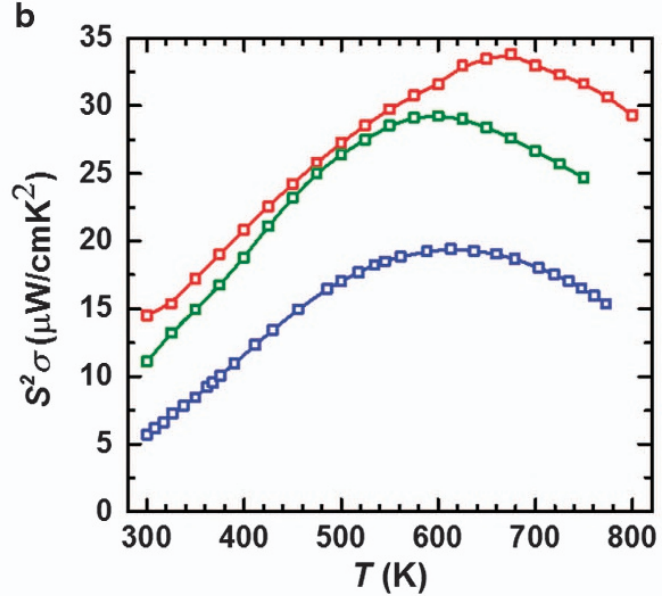

d

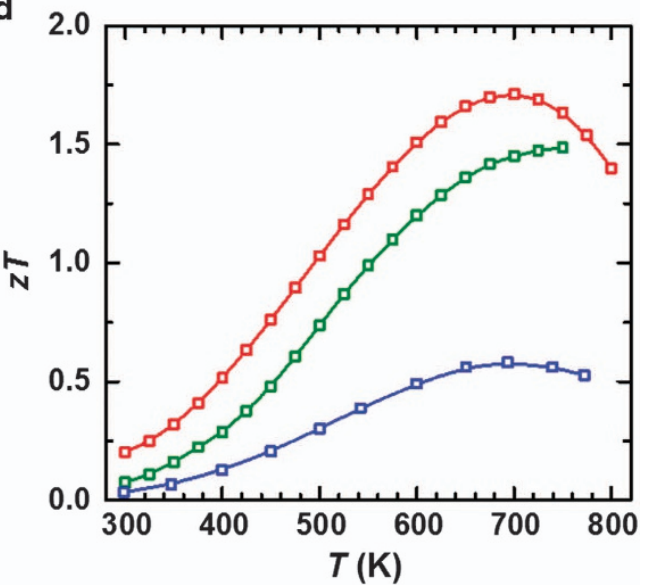

Figure 6 Comparison on the temperature-dependent Seebeck coefficient (a), power factor (b), total and lattice thermal conductivity (c) and figure of merit (d) for $\mathrm{SnTe}: \mathrm{I}^{37} \mathrm{PbTe}: \mathrm{Na}^{36}$ and $\mathrm{GeTe}: \mathrm{Bi}$, showing the highest $z T$ by hole concentration optimization only.

that face-centered cubic-structured $\beta$-GeTe, $\mathrm{PbTe}$ and $\mathrm{SnTe}$ are all direct-gap semiconductors with the first valence band edge located at the $L$ point, whereas the second one is along the $\Sigma$ line, as shown in Figures $2 \mathrm{~b}-\mathrm{d}$, respectively. A similar two-valence band configuration can be seen in the low-temperature rhombohedral phase $(\alpha)$. The most distinguishing feature for $\alpha-G e T e$ is the switch in energy levels between the $L$ and $\Sigma$ bands, as displayed in Figure 2a, meaning a higher energy of the $\Sigma$ band than that of the $L$ band. The much heavier effective mass of the $\Sigma$ band nicely explains the much higher carrier concentration ${ }^{80}$ required in GeTe for its first peak in the power factor, as shown in Figure 1. The unique $\Sigma$ band dominant carrier transport in rhombohedral GeTe, along with the smallest effective mass for an individual valence band (Table 1), should lead to its TE properties being significantly different from those of cubic SnTe and $\mathrm{PbTe}$, as will be discussed in detail.

We first achieved a broad range of carrier concentrations by both intrinsic $\mathrm{Ge}$ doping $\left(\mathrm{Ge}_{1+\delta} \mathrm{Te},-0.04 \leqslant \delta<0.06\right)$ and extrinsic Bi doping at $\mathrm{Ge}$ sites $\left(\mathrm{Ge}_{1-} \mathrm{Bi}_{x} \mathrm{Te}, x \leqslant 0.1\right)$, showing a linear decrease with $x$ increasing up to 0.07 and saturation afterwards (Figure $3 \mathrm{a}$ ). In the case of intrinsic Ge doping ( $\delta>0$ for Ge excess and $\delta<0$ for Ge deficiency), the carrier concentration saturates at $7 \times 10^{20} \mathrm{~cm}^{-3}$ on the Ge excess side $(\delta=0.02)$ and at $2.3 \times 10^{21} \mathrm{~cm}^{-3}$ on the Ge deficiency side $(\delta=-0.04)$. The composition and the carrier concentration for the samples are listed in Supplementary Table 1. The obtained broad range of the carrier concentration not only enables a meaningful discussion of the transport properties but also the estimation of the optimal range for the TE properties. The comparison of the temperature-dependent Hall mobility $\left(\mu_{\mathrm{H}}\right.$; Figure $3 \mathrm{~b}$ ) for these three compounds shows an unchanged dominant carrier scattering by acoustic phonons, as $\mu_{\mathrm{H}}$ rapidly decreases with the increasing $T$. This validates the exclusion of the effects on the transport properties due to carrier scattering. It should be noted that the Hall mobility decreases much faster at high temperatures $(T>\sim 650 \mathrm{~K})$ than what acoustic scattering predicts. This is presumably due to the bipolar conduction, as well as the redistribution of carriers between the two valence bands with significantly different mobilities.

It is then straightforward that the unique dominant heavy $\Sigma$ band leads to the highest Seebeck coefficient in rhombohedral GeTe ( $\alpha$ phase) compared with the other two compounds at the carrier concentrations $\left(<10^{21} \mathrm{~cm}^{-3}\right)$ experimentally achievable so far. According to the two-valence band structure with acoustic scattering, the band parameters listed in Table 1 enable a nice prediction (red curve in Figure 3c) of the carrier concentration-dependent Seebeck coefficient at room temperature, indicating the validity of a rigid band approximation in this work. The model prediction assumes deformation potential coefficients of 9.5 and $23.5 \mathrm{eV}$ for the $\Sigma$ and $L$ bands, respectively. It should be noted that in the optimal carrier concentration range of $1-3 \times 10^{20} \mathrm{~cm}^{-3}$, the transport of carriers in $\alpha$-GeTe at low temperatures has negligible contributions from the lower $L$ band owing to the fact that most of the carriers are within the 
$\Sigma$ band. This is reflected by the calculated relationship between the Fermi level and the carrier concentration in Figure 2a.

Owing to the heavy effective mass (of the $\Sigma$ band) and the high concentration of dopants, $\alpha$-GeTe has the lowest mobility (Figure 3d) at room temperature. In addition to carrier scattering by dopant impurities, ${ }^{81}$ the further reduction in the Hall mobility at high Bi-doping levels (low hole concentrations, $<\sim 2 \times 10^{20} \mathrm{~cm}^{-3}$ ) in $\mathrm{GeTe}$ is presumably due to the redistribution of carriers from the high-mobility $L$ band to the low-mobility $\Sigma$ band. Such a redistribution is normally seen in similar materials including $\mathrm{PbTe},{ }^{19,23,82}$ $\mathrm{SnTe}^{83}$ and $\mathrm{PbSe}^{27}$

When the temperature increases, GeTe undergoes a phase transition from a rhombohedral structure ( $\alpha$ phase) to one that is cubic ( $\beta$ phase), leading to a switch in energy between the $L$ and $\Sigma$ bands. This indicates that a dominant heavy $\Sigma$ band conduction at low temperatures transits to a conduction contributed by both the $L$ and $\Sigma$ bands at high temperatures (Figure 2). This band switch results in the redistribution of large population carriers from the heavy $\Sigma$ to the light $L$ band in the high-temperature phase. Both the resistivity and the Seebeck coefficient (or, more specifically, the slope of $S$ vs $T$ ) are expected to be reduced because of the much higher mobility and the much smaller density of state effective mass of the light $L$ band. All of these expectations are well supported by the experimental measurements, and an example is given in Figure 4 showing the resistivity and the Seebeck coefficient in the vicinity of the phase transition temperature for high- $z T$ samples with a room temperature $n_{\mathrm{H}}=1.37 \times 10^{20} \mathrm{~cm}^{-3}$. The evolution of the Fermi surface during the phase transition is also shown. This phenomenon has rarely been reported, but it is very helpful for understanding the mechanism and therefore for guiding the further improvement of the TE performance in this material.

The temperature-dependent resistivity, Seebeck coefficient, thermal conductivity and figure of merit $z T$ for $\mathrm{Bi}_{x} \mathrm{Ge}_{1-x} \mathrm{Te}$ are shown in Figures $5 \mathrm{a}-\mathrm{d}$. The majority of the samples studied here show a degenerated semiconducting behavior with an observable change in the phase transition region. The decrease in both the Seebeck coefficient Figure $5 \mathrm{a}$ and resistivity Figure $5 \mathrm{~b}$ at high temperatures $(T>\sim 700 \mathrm{~K})$ for samples with $n_{\mathrm{H}} \leqslant 3.5 \times 10^{20} \mathrm{~cm}^{-3}$ is a good indication of mixed conduction by both the majority holes and the thermally excited minority electrons. The lattice thermal conductivity (inset of Figure 5c) is obtained by subtracting the electronic contribution $\left(\kappa_{\mathrm{e}}=L T / \rho\right)$ from the total thermal conductivity Figure $5 c$, where the Lorenz factor ( $L$; Supplementary Figure 3 ) is derived based on a single Kane band model approximation. The fact that the lower carrier concentration leads to a more obvious change in the transport properties can be well understood by the resulting less degenerated Fermi level that better probes the character of the first valence band edge in different phases.

The figure of merit, $z T$, shown in Figure $5 \mathrm{~d}$ is high in both the lowand high-temperature phases, showing a peak value of up to $\sim 1.8$. This is actually the highest among the three group IV monotellurides, as evidenced from Figure 6, where detailed temperature-dependent properties including the Seebeck coefficient (Figure $6 a$ ), power factor (Figure $6 \mathrm{~b}$ ), thermal conductivity (Figure $6 \mathrm{c}$ ) and $z T$ (Figure $6 \mathrm{~d}$ ) are included for a comparison among the three pristine compounds with respective optimal carrier concentrations.

To reveal the electronic origins of the observed superior TE performance of GeTe, it is known that the maximal power factor is proportional to the number of degenerated band valleys $\left(N_{\mathrm{v}}\right)$ and inversely proportional to the inertial mass $\left(m_{\mathrm{I}}^{*}\right)$ of each valley $\left(P F \propto \mu m^{* 3 / 2} \propto N_{\mathrm{v}} / m_{\mathrm{I}}^{*}\right)$ when the scattering of carriers is dominated by acoustic phonons, ${ }^{26,82,84,85}$ as is the case in the group IV monotellurides studied here. This indicates that a large $N_{\mathrm{v}}$ and a small $m_{\mathrm{I}}^{*}$ are beneficial for TE $s$.

Comparing the band effective masses of PbTe and GeTe in the cubic phase, it is seen that the $L$ and $\Sigma$ bands are $\sim 20 \%$ and $40 \%$ lighter, respectively, in the latter (Table 1). This difference explains the higher power factor of GeTe by $\sim 30 \%$ at $T>\sim 650 \mathrm{~K}$ (Figure $6 \mathrm{~b}$ ) without involving the complexity of the temperature-dependent energy offset between these two bands in these compounds. ${ }^{82,86,87}$ Therefore, the lower effective mass can be considered the main reason for the superior TE performance in cubic GeTe (at high temperatures). For low temperatures, because of the overall high valley degeneracy $\left(N_{\mathrm{v}}\right)$ with a sufficiently low effective mass (Table 1) for the dominant $\Sigma$ valence band in rhombohedral GeTe (Figure 2), the power factor remains slightly higher. It should be noted that the lower lattice thermal conductivity (Figure $6 \mathrm{c}$ ) contributes to the high $z T$ in GeTe at low temperatures $(T<\sim 600 \mathrm{~K})$ as well. As a whole, both low- and high-temperature phase GeTe obtain electronic advantages from their favorable band structures for superior TE performance.

\section{CONCLUSION}

By comparing GeTe with $\mathrm{PbTe}$ and $\mathrm{SnTe}$, it is concluded that the superior TE performance of GeTe mainly stems from its favorable energy band structure. The lower effective mass of both the $L$ band and $\Sigma$ bands at high temperatures and the dominance of the highdegeneracy $\Sigma$ band conduction at low temperatures, as the main electronic origin, guarantee a high power factor in GeTe. The large number of samples synthesized here with a broad range of hole concentrations enables a meaningful understanding of the transport properties inherent to this material, setting up a reference substance for both guiding and evaluating further improvements by other proven strategies. The obtained high $z T$ inherent to this compound in the entire temperature range demonstrates that GeTe itself is an excellent matrix material for realizing extraordinary TE performance.

\section{CONFLICT OF INTEREST}

The authors declare no conflict of interest.

\section{ACKNOWLEDGEMENTS}

This work is supported by the National Natural Science Foundation of China (Grant Nos 51422208, 11474219, 11674211 and 51401147), the National Recruitment Program of Global Youth Experts (1000 Plan), the Fundamental Research Funds for the Central Universities and the Bayer-Tongji EcoConstruction and Material Academy (TB20140001). JY acknowledges the support from the Program for Professor of Special Appointment (Eastern Scholar) at the Shanghai Institutions of Higher Learning (TP2015041).

1 Bell, L. E. Cooling, heating, generating power, and recovering waste heat with thermoelectric systems. Science 321, 1457-1461 (2008).

2 Hsu, K. F., Loo, S., Guo, F., Chen, W., Dyck, J. S., Uher, C., Hogan, T., Polychroniadis, E. K. \& Kanatzidis, M. G. Cubic AgPb $\mathrm{SbTe}_{2+m}$ : Bulk thermoelectric materials with high figure of merit. Science 303, 818-821 (2004).

3 Biswas, K., He, J., Blum, I. D., Wu, C.-I., Hogan, T. P., Seidman, D. N., Dravid, V. P. \& Kanatzidis, M. G. High-performance bulk thermoelectrics with all-scale hierarchical architectures. Nature 489, 414-418 (2012).

4 Pei, Y., Lensch-Falk, J., Toberer, E. S., Medlin, D. L. \& Snyder, G. J. High thermoelectric performance in $\mathrm{PbTe}$ due to large nanoscale Ag2Te precipitates and La doping. Adv. Funct. Mater. 21, 241-249 (2011).

5 Sang II Kim, K. H. L., Lee, K. H., Mun, H. A., Kim, H. S., Hwang, S. W., Roh, J. W., Yang, D. J., Shin, W. H., Li, X. S., Lee, Y. H., Snyder, G. J. \& Kim, S. W. Thermoelectrics. Dense dislocation arrays embedded in grain boundaries for high-performance bulk thermoelectrics. Science 348, 109-114 (2015).

6 Poudel, B., Hao, Q., Ma, Y., Lan, Y. C., Minnich, A., Yu, B., Yan, X. A., Wang, D. Z., Muto, A., Vashaee, D., Chen, X. Y., Liu, J. M., Dresselhaus, M. S., Chen, G. \& Ren, Z. F. 
High-thermoelectric performance of nanostructured bismuth antimony telluride bulk alloys. Science 320, 634-638 (2008).

7 Zhao, W., Liu, Z., Wei, P., Zhang, Q., Zhu, W., Su, X., Tang, X., Yang, J., Liu, Y., Shi, J., Chao, Y., Lin, S. \& Pei, Y. Magnetoelectric interaction and transport behaviours in magnetic nanocomposite thermoelectric materials. Nat. Nanotechnol. 12, 55-60 (2016).

8 Zhao, L. D., Lo, S. H., Zhang, Y., Sun, H., Tan, G., Uher, C., Wolverton, C., Dravid, V. P. \& Kanatzidis, M. G. Ultralow thermal conductivity and high thermoelectric figure of merit in SnSe crystals. Nature 508, 373-377 (2014).

9 Morelli, D. T., Jovovic, V. \& Heremans, J. P. Intrinsically minimal thermal conductivity in cubic I-V-VI 2 semiconductors. Phys. Rev. Lett. 101, 035901 (2008).

10 Liu, H., Shi, X., Xu, F., Zhang, L. \& Zhang, W. Copper ion liquid-like thermoelectrics. Nat. Mater. 11, 422-425 (2012).

11 Qiu, W., Xi, L., Wei, P., Ke, X., Yang, J. \& Zhang, W. Part-crystalline part-liquid state and rattling-like thermal damping in materials with chemical-bond hierarchy. Proc. Nat Acad. Sci. USA 111, 15031-15035 (2014).

12 Pei, Y. \& Morelli, D. Vacancy phonon scattering in thermoelectric $\operatorname{In}_{2} T e_{3}-\operatorname{InSb}$ solid solutions. Appl. Phys. Lett. 94, 122112 (2009).

13 Shen, J., Zhang, X., Lin, S., Li, J., Chen, Z., Li, W. \& Pei, Y. Vacancy scattering for enhancing the thermoelectric performance of $\mathrm{CuGaTe}_{2}$ solid solutions. J. Mater. Chem. A 4, 15464-15470 (2016).

14 Chen, Z., Ge, B., Li, W., Lin, S., Shen, J., Chang, Y., Hanus, R., Snyder, G. J. \& Pei, Y. Vacancy-induced dislocations within grains for high-performance PbSe thermoelectrics. Nat. Commun. 8, 13828 (2017).

15 Pei, Y., Zheng, L., Li, W., Lin, S., Chen, Z., Wang, Y., Xu, X., Yu, H., Chen, Y. \& Ge, B. Interstitial point defect scattering contributing to high thermoelectric performance in SnTe. Adv. Electron. Mater. 2, 1600019 (2016).

16 Li, W., Lin, S., Ge, B., Yang, J., Zhang, W. \& Pei, Y. Low sound velocity contributing to the high thermoelectric performance of Ag8SnSe6. Adv. Sci. 3 1600196 (2016).

17 Pei, Y., Shi, X., Lalonde, A., Wang, H., Chen, L. \& Snyder, G. J. Convergence of electronic bands for high performance bulk thermoelectrics. Nature 473, 66-69 (2011).

18 Liu, W., Tan, X., Yin, K., Liu, H., Tang, X., Shi, J., Zhang, Q. \& Uher, C. Convergence of conduction bands as a means of enhancing thermoelectric performance of n-type $\mathrm{Mg}_{2} \mathrm{Si}_{1-x} \mathrm{Sn}_{x}$ solid solutions. Phys. Rev. Lett. 108, 166601 (2012).

19 Pei, Y., Lalonde, A. D., Heinz, N. A., Shi, X., Iwanaga, S., Wang, H., Chen, L. \& Snyder, G. J. Stabilizing the optimal carrier concentration for high thermoelectric efficiency. Adv. Mater. 23, 5674-5678 (2011).

20 Xie, H., Wang, H., Pei, Y., Fu, C., Liu, X., Snyder, G. J., Zhao, X. \& Zhu, T. Beneficial contribution of alloy disorder to electron and phonon transport in half-Heusler thermoelectric materials. Adv. Funct. Mater. 23, 5123-5130 (2013).

21 Pei, Y., Lalonde, A. D., Heinz, N. A. \& Snyder, G. J. High thermoelectric figure of merit in $\mathrm{PbTe}$ alloys demonstrated in $\mathrm{PbTe}-\mathrm{CdTe}$. Adv. Energy Mater. 2, 670-675 (2012).

22 Zhang, Q., Cao, F., Liu, W., Lukas, K., Yu, B., Chen, S., Opeil, C., Broido, D., Chen, G. \& Ren, Z. Heavy doping and band engineering by potassium to improve the thermoelectric figure of merit in p-type PbTe, PbSe, and $\mathrm{PbTe}_{1-y} \mathrm{Se}_{y}$. J. Am. Chem. Soc. 134, 10031-10038 (2012)

23 Pei, Y., Wang, H., Gibbs, Z. M., Lalonde, A. D. \& Snyder, G. J. Thermopower enhancement in $\mathrm{Pb}_{1-x} \mathrm{Mn}_{x}$ Te alloys and its effect on thermoelectric efficiency. NPG Asia Mater. 4, 1-6 (2012).

24 Jian, Z., Chen, Z., Li, W., Yang, J., Zhang, W. \& Pei, Y. Significant band engineering effect of YbTe for high performance thermoelectric PbTe. J. Mater. Chem. C 3, 12410-12417 (2015).

25 Lin, S., Li, W., Chen, Z., Shen, J., Ge, B. \& Pei, Y. Tellurium as a high-performance elemental thermoelectric. Nat. Commun. 7, 10287 (2016).

26 Pei, Y., Lalonde, A. D., Wang, H. \& Snyder, G. J. Low effective mass leading to high thermoelectric performance. Energy Environ. Sci. 5, 7963-7969 (2012).

27 Wang, H., Pei, Y., Lalonde, A. D. \& Snyder, G. J. Weak electron-phonon coupling contributing to high thermoelectric performance in n-type PbSe. Proc. Natl Acad. Sci. USA 109, 9705-9709 (2012).

28 Herman, F., Kortum, R. L., Ortenburger, I. B. \& Van Dyke, J. P. Relativistic band struture of GeTe, SnTe, PbTe, PbSe and PbS. J. Phys. Colloq. 29, 62-77 (1968)

29 Sitter, H., Lischka, K. \& Heinrich, H. Structure of the second valence band in $\mathrm{PbTe}$. Phys. Rev. B 16, 680-687 (1977).

30 Allgaier, R. S. Valence bands in lead telluride. J. Appl. Phys. 32, 2185-2189 (1961).

31 Brebrick, R. F. \& Strauss, A. J. Anomalous thermoelectric power as evidence for two-valence bands in SnTe. Phys. Rev. 131, 104-110 (1963).

32 Rogers, L. M. Valence band structure of SnTe. J. Appl. Phys. 1, 845-852 (1968).

33 Gonzalezt, P., Agapito, J. A. \& Pardo, D. Two-band model parameters deduced from Hall coefficient measurements in polycrystalline films of SnTe. J. Phys. C 19, 899-907 (1986).

34 Santhanam, S. \& Chaudhuri, A. K. Transport properties of SnTe interpreted by means of a two valence band model. Mater. Res. Bull 16, 911-917 (1981).

35 Crocker, A. J. \& Rogers, L. M. Valence band structure of PbTe. J. Phys. Coll. 29: C-129-C-132 (1968)

36 Pei, Y., Lalonde, A., Iwanaga, S. \& Snyder, G. J. High thermoelectric figure of merit in heavy-hole dominated PbTe. Energy Environ. Sci. 4, 2085-2089 (2011).

37 Zhou, M., Gibbs, Z. M., Wang, H., Han, Y., Xin, C., Li, L. \& Snyder, G. J. Optimization of thermoelectric efficiency in SnTe: the case for the light band. Phys. Chem. Chem. Phys. 16, 20741-20748 (2014)
38 Wu, D., Zhao, L. D., Hao, S. Q., Jiang, Q. K., Zheng, F. S., Doak, J. W., Wu, H. J., Chi, H., Gelbstein, Y., Uher, C., Wolverton, C., Kanatzidis, M. \& He, J. Q. Origin of the high performance in GeTe-based thermoelectric materials upon $\mathrm{Bi}_{2} \mathrm{Te}_{3}$ doping. J. Am. Chem. Soc. 136, 11412-11419 (2014).

39 Perumal, S., Roychowdhury, S., Negi, D. S., Datta, R. \& Biswas, K. High thermoelectric performance and enhanced mechanical stability of p-type $\mathrm{Ge}_{1-x} \mathrm{Sb}_{x} \mathrm{Te}$. Chem. Mater. 27, 7171-7178 (2015).

40 Sun, H., Lu, X., Chi, H., Morelli, D. T. \& Uher, C. Highly efficient $\left(\ln _{2} T e_{3}\right)_{x}(G e T e)_{3}-3 x$ thermoelectric materials: a substitute for TAGS. Phys. Chem. Chem. Phys. 16, 15570-15575 (2014)

41 Lee, J. K., Oh, M. W., Kim, B. S., Min, B. K., Lee, H. W. \& Park, S. D. Influence of Mn on crystal structure and thermoelectric properties of GeTe compounds. Electron. Mater. Lett. 10, 813-817 (2014).

42 Ch., G., Christakudis, S. K. P., Shelimova, L. E. \& Avilov, E. S. Thermoelectric figure of merit of some compositions in the system $(\mathrm{GeTe})_{1-x}\left[(\mathrm{Ag} 2 \mathrm{Te})_{1-y}(\mathrm{Sb} 2 \mathrm{Te} 3)_{y}\right]_{x}$. Phys. Stat. Sol. (a) 128, 465-471 (1991).

43 Plachkova, S. K. Thermoelectric figure of merit of the system $(\mathrm{GeTe})_{1-x}(\mathrm{AgSbTe} 2)_{x}$ Phys. Stat. Sol. (a) 83, 349-355 (1984)

44 Levin, E. M., Cook, B. A., Harringa, J. L., Bud'ko, S. L., Venkatasubramanian, R. \& Schmidt-Rohr, K. Analysis of Ce- and Yb-doped TAGS-85 materials with enhanced thermoelectric figure of merit. Adv. Funct. Mater. 21, 441-447 (2011).

45 Levin, E. M., Bud'ko, S. L. \& Schmidt-Rohr, K. Enhancement of thermopower of TAGS-85 high-performance thermoelectric material by doping with the rare earth Dy. Adv. Funct. Mater. 22, 2766-2774 (2012).

46 Rosenthal, T., Schneider, M. N., Stiewe, C., Döblinger, M. \& Oeckler, O. Real structure and thermoelectric properties of GeTe-rich germanium antimony tellurides. Chem. Mater. 23, 4349-4356 (2011).

47 Fahrnbauer, F., Souchay, D., Wagner, G. \& Oeckler, O. High thermoelectric figure of merit values of germanium antimony tellurides with kinetically stable cobalt Germanide precipitates. J. Am. Chem. Soc. 137, 12633-12638 (2015).

48 Rosenthal, T., Urban, P., Nimmrich, K., Schenk, L., De Boor, J., Stiewe, C. \& Oeckler, O. Enhancing the thermoelectric properties of germanium antimony tellurides by substitution with Selenium in compounds $\mathrm{Ge}_{n} \mathrm{Sb}_{2}\left(\mathrm{Te}_{1-x} \mathrm{Se}_{\chi}\right)_{n+3}$ $(0 \leqslant x \leqslant 0.5 ; n \geq 7)$. Chem. Mater. 26, 2567-2578 (2014).

49 Davidow, J. \& Gelbstein, Y. A comparison between the mechanical and thermoelectric properties of three highly efficient p-type GeTe-rich compositions: TAGS-80, TAGS-85, and $3 \% \mathrm{Bi}_{2} \mathrm{Te}_{3}$-doped $\mathrm{Ge}_{0.87} \mathrm{~Pb}_{0.13} \mathrm{Te}$. J. Electron. Mater. 42 1542-1549 (2013).

50 Gelbstein, Y., Ben-Yehuda, O., Pinhas, E., Edrei, T., Sadia, Y., Dashevsky, Z. \& Dariel, M. P. Thermoelectric properties of $(\mathrm{Pb}, \mathrm{Sn}, \mathrm{Ge})$ Te-based alloys. J. Electron. Mater. 38, 1478-1482 (2009).

51 Hazan, E., Madar, N., Parag, M., Casian, V., Ben-Yehuda, O. \& Gelbstein, Y. Effective electronic mechanisms for optimizing the thermoelectric properties of GeTe-rich alloys. Adv. Electron. Mater. 1, 1500228 (2015).

52 Wiedemeier, H. \& Siemers, P. A. in Modern High Temperature Sciences: A Collection of Research Papers from Scientists, Post-Doctoral Associates, and Colleagues of Professor Leo Brewer in celebration of his 65th Birthday (ed. Margrave, J. L.) (Humana Press, New York, NY, USA, 1984).

53 Gelbstein, Y., Dado, B., Ben-Yehuda, O., Sadia, Y., Dashevsky, Z. \& Dariel, M. Highly efficient Ge-rich $\mathrm{Ge}_{x} \mathrm{~Pb}_{1-\mathrm{x}} \mathrm{Te}$ thermoelectric alloys. J. Electron. Mater. 39, 2049-2052 (2010)

54 Gelbstein, Y., Davidow, J., Girard, S. N., Chung, D. Y. \& Kanatzidis, M. Controlling metallurgical phase separation reactions of the $\mathrm{Ge}_{0.87} \mathrm{~Pb}_{0.13} \mathrm{Te}$ alloy for high thermoelectric performance. Adv. Energy Mater. 3, 815-820 (2013).

55 Gelbstein, Y. \& Davidow, J. Highly efficient functional $\mathrm{Ge}_{x} \mathrm{~Pb}_{1-x} \mathrm{Te}$ based thermoelectric alloys. Phys. Chem. Chem. Phys. 16, 20120-20126 (2014).

56 Li, J. Q., Deng, J. F., Li, S. K., Li, Y., Liu, F. S. \& Ao, W. Q. Phases and thermoelectric properties of $\mathrm{Ge}_{1-x}\left(\mathrm{~Pb}_{0.9} \mathrm{Yb}_{0.1}\right)_{x} \mathrm{Te}$ alloys. Intermetallics 56, 63-67 (2015).

57 Gelbstein, Y., Dado, B., Ben-Yehuda, O., Sadia, Y., Dashevsky, Z. \& Dariel, M. P. High thermoelectric figure of merit and nanostructuring in bulk p-type $\mathrm{Ge}_{x}\left(\mathrm{SnyPb}_{1-y}\right)_{1-x} \mathrm{Te}$ alloys following a spinodal decomposition reaction. Chem. Mater. 22, 1054-1058 (2010).

58 Gelbstein, Y., Dashevsky, Z. \& Dariel, M. P. Highly efficient bismuth telluride doped p-type $\mathrm{Pb}_{0.13} \mathrm{Ge}_{0.87} \mathrm{Te}$ for thermoelectric applications. Phys. Stat. Sol. 1, 232-234 (2007).

59 Chattopadhyay, T., Boucherle, J. X. \& von Schnering, H. G. Neutron diffraction study on the structural phase transition in GeTe. J. Phys. C 20, 1431-1440 (1987).

60 Gelbstein, Y., Ben-Yehuda, O., Dashevsky, Z. \& Dariel, M. P. Phase transitions of p-type $(\mathrm{Pb}, \mathrm{Sn}, \mathrm{Ge}) \mathrm{Te}$-based alloys for thermoelectric applications. J. Cryst. Growth 311, 4289-4292 (2009).

61 Okamoto, H. Ge-Te (germanium-tellurium). J. Phase Equilib. 21, 496-496 (2000).

62 Pei, Y., Wang, H. \& Snyder, G. J. Band engineering of thermoelectric materials. Adv. Mater. 24, 6125-6135 (2012).

63 Li, W., Chen, Z., Lin, S., Chang, Y., Ge, B., Chen, Y. \& Pei, Y. Band and scattering tuning for high performance thermoelectric $\mathrm{Sn}_{1-x} \mathrm{Mn}_{x}$ Te alloys. J. Materiomics 1, 307-315 (2015).

64 Zhang, Q., Liao, B., Lan, Y., Lukas, K., Liu, W., Esfarjani, K., Opeil, C., Broido, D. Chen, G. \& Ren, Z. High thermoelectric performance by resonant dopant indium in nanostructured SnTe. Proc. Natl Acad. Sci. USA 110, 13261-13266 (2013).

65 Damon, D. H., Lubell, M. S. \& Mazelsky, R. Nature of the defect in germanium telluride. J. Phys. Chem. Solids. 28, 520-522 (1966)

66 Lewis, J. E. The defect structure of non-stoichiometric germanium telluride from magnetic susceptibility measurements. Phys. Stat. Sol. 38, 131-140 (1970). 
67 Lalonde, A. D., Ikeda, T. \& Snyder, G. J. Rapid consolidation of powdered materials by induction hot pressing. Rev. Sci. Instrum. 82, 025104 (2011).

68 Zhou, Z. \& Uher, C. Apparatus for Seebeck coefficient and electrical resistivity measurements of bulk thermoelectric materials at high temperature. Rev. Sci. Instrum. 76, 023901 (2005).

69 Welzmiller, S., Fahrnbauer, F., Hennersdorf, F., Dittmann, S., Liebau, M., Fraunhofer, C., Zeier, W. G., Snyder, G. J. \& Oeckler, O. Increasing Seebeck coefficients and thermoelectric performance of $\mathrm{Sn} / \mathrm{Sb} / \mathrm{Te}$ and $\mathrm{Ge} / \mathrm{Sb} / \mathrm{Te}$ materials by $\mathrm{Cd}$ doping. Adv. Electron. Mater. 1, 1500266 (2015).

$70 \mathrm{Li}$, J., Zhang, X., Lin, S., Chen, Z. \& Pei, Y. Realizing the high thermoelectric performance of GeTe by Sb-doping and Se-alloying. Chem. Mater. 29, 605-611 (2016).

71 Cardona, M. \& Greenaway, D. L. Optical properties and band structure of group IV-VI and group V materials. Phys. Rev. 133, A1685-A1697 (1964).

72 Lewis, J. E. Optical properties and energy gap of GeTe from reflectance studies. Phys. Stat. Sol. (b) 59, 367-377 (1973).

73 Tung, Y. W. \& Cohen, M. L. Relativistic band structure and electronic properties of $\mathrm{SnTe}, \mathrm{GeTe}$, and PbTe. Phys. Rev. 180, 823-826 (1969).

74 Korzhuev, M. A. On the fundermental energy gap in GeTe. Phys. Stat. Sol. (b) 112 , K39 (1982).

75 Okoye, C. M. I. Electronic and optical properties of SnTe and GeTe. J. Phys. Condens. Matter 14, 8625-8637 (2002)

76 Di Sante, D., Barone, P., Bertacco, R. \& Picozzi, S. Electric control of the giant Rashba effect in bulk GeTe. Adv. Mater. 25, 509-513 (2013).

77 Singh, D. J. Optical properties of cubic and rhombohedral GeTe. J. Appl. Phys. 113, 203101 (2013).

78 Liebmann, M., Rinaldi, C., Di Sante, D., Kellner, J., Pauly, C., Wang, R. N. Boschker, J. E., Giussani, A., Bertoli, S., Cantoni, M., Baldrati, L., Asa, M., Vobornik, I., Panaccione, G., Marchenko, D., Sanchez-Barriga, J., Rader, O., Calarco, R., Picozzi, S., Bertacco, R. \& Morgenstern, M. Giant Rashba-type spin splitting in ferroelectric GeTe (111). Adv. Mater. 28, 560-565 (2016).

79 Lewis, J. E. Band sturcture and nature of lattice defects in GeTe from analysize of electrical properties. Phys. Stat. Sol. 36, 737-745 (1969).

80 Pei, Y., Gibbs, Z. M., Balke, B., Zeier, W. G. \& Snyder, G. J. Optimum carrier concentration in n-type PbTe thermoelectrics. Adv. Energy Mater. 4, 1400486 (2014).

81 Wang, H., Lalonde, A. D., Pei, Y. \& Snyder, G. J. The criteria for beneficial disorder in thermoelectric solid solutions. Adv. Funct. Mater. 23, 1586-1596 (2013).
82 Ravich, Y. I., Efimova, B. A. \& Smirnov, I. A. Semiconducting Lead Chalcogenides (Plenum Press, New York, NY, USA, 1970).

83 Chaudhuri, A. K. \& Santhanam, S. Transport properties of SnTe interpreted by means of a two valence band model. Mater. Res. Bull. 16, 911-917 (1981).

84 Heikes, R. R. Thermoelectricity: Science and Engineering (eds Heikes, R. R. \& Ure, R. W.) (Interscience Publisher, New York, NY, USA, 1961).

85 Chasmar, R. P. \& Stratton, R. The thermoelectric figure of merit and its relation to thermoelectric generators. J. Electron. Contr. 7, 52-72 (1959).

86 Sysoeva, L. M., Lev, E. Y. \& Kolomoets, N. V. Influence of the second valence band and of the conduction band on the thermoelectric figure of merit of germanium telluride. Sov. Phys. Semicond. 3, 1099-1102 (1970).

87 Tauber, R. N., Machonis, A. A. \& Cadoff, I. B. Thermal and optical energy gaps in PbTe. J. Appl. Phys. 37, 4855-4860 (1966).

88 Nashchekina, O. N., Rogacheva, E. I. \& Vodorez, O. S. Influence of vanadium on the defect structure and thermoelectric properties of GeTe. J. Electron. Mater. 42, 1771-1775 (2013).

89 Erasova, N. A., Kaidanov, V. I., Chernik, I. A., Sysoeva, L. M., Lev, E. Ya. \& Kolomoets, N. V. Electronic properties of GeTe at low temperature. Sov. Phys.-Semicond 3, 1289-1293 (1970).

90 Korzhuev, M. A. \& Low, L. I. P. Temperature thermoelectricpower of GeTe. Fiz. Tekh. Poluprovodn. 16, 1296-1298 (1982).

(c) (i) This work is licensed under a Creative Commons Attribution 4.0 International License. The images or other third party material in this article are included in the article's Creative Commons license, unless indicated otherwise in the credit line; if the material is not included under the Creative Commons license, users will need to obtain permission from the license holder to reproduce the material. To view a copy of this license, visit http:// creativecommons.org/licenses/by/4.0/

(C) The Author(s) 2017

Supplementary Information accompanies the paper on the NPG Asia Materials website (http://www.nature.com/am) 